\title{
Effect of Cinacalcet on Cardiovascular Disease in Patients Undergoing Dialysis
}

\author{
The EVOLVE Trial Investigators*
}

\begin{abstract}
Members of the writing committee are listed in the Appendix. Address reprint requests to Dr. Glenn M. Chertow at Stanford University School of Medicine, 780 Welch Rd., Suite 106, Palo Alto, CA 93034, or at gchertow@stanford.edu.

* Members of the Evaluation of Cinacalcet Hydrochloride Therapy to Lowe Cardiovascular Events (EVOLVE) Tria Group are listed in the Supplementary Appendix, available at NEJM.org.
\end{abstract}

This article was published on November 3, 2012, at NEJM.org.

N EnglJ Med 2012;367:2482-94. DOI: 10.1056/NEJMoal205624

Copyright (؟ 2012 Massachusetts Medical Society.

\section{A B STRACT}

\section{BACKGROUND}

Disorders of mineral metabolism, including secondary hyperparathyroidism, are thought to contribute to extraskeletal (including vascular) calcification among patients with chronic kidney disease. It has been hypothesized that treatment with the calcimimetic agent cinacalcet might reduce the risk of death or nonfatal cardiovascular events in such patients.

\section{METHODS}

In this clinical trial, we randomly assigned 3883 patients with moderate-to-severe secondary hyperparathyroidism (median level of intact parathyroid hormone, 693 pg per milliliter [10th to 90th percentile, 363 to 1694]) who were undergoing hemodialysis to receive either cinacalcet or placebo. All patients were eligible to receive conventional therapy, including phosphate binders, vitamin $\mathrm{D}$ sterols, or both. The patients were followed for up to 64 months. The primary composite end point was the time until death, myocardial infarction, hospitalization for unstable angina, heart failure, or a peripheral vascular event. The primary analysis was performed on the basis of the intention-to-treat principle.

\section{RESULTS}

The median duration of study-drug exposure was 21.2 months in the cinacalcet group, versus 17.5 months in the placebo group. The primary composite end point was reached in 938 of 1948 patients (48.2\%) in the cinacalcet group and 952 of 1935 patients (49.2\%) in the placebo group (relative hazard in the cinacalcet group vs. the placebo group, 0.93 ; $95 \%$ confidence interval, 0.85 to $1.02 ; \mathrm{P}=0.11$ ). Hypocalcemia and gastrointestinal adverse events were significantly more frequent in patients receiving cinacalcet.

\section{CONCLUSIONS}

In an unadjusted intention-to-treat analysis, cinacalcet did not significantly reduce the risk of death or major cardiovascular events in patients with moderate-to-severe secondary hyperparathyroidism who were undergoing dialysis. (Funded by Amgen; EVOLVE ClinicalTrials.gov number, NCT00345839.) 
ARDIOVASCULAR DISEASE IS VERY COMmon among patients with chronic kidney disease, including those treated with hemodialysis, among whom the risk of death from cardiovascular disease is increased by a factor of 10 or more as compared with the risk in the general population. ${ }^{1,2}$ Cardiovascular risk factors that have been linked to chronic kidney disease include heightened states of inflammation, ${ }^{3}$ oxidative stress, ${ }^{4}$ activation of the renin-angiotensinaldosterone system ${ }^{5}$ and the sympathetic nervous system, ${ }^{6}$ endothelial dysfunction, ${ }^{7}$ retention of uremic toxins promoting atherosclerosis and arteriosclerosis, ${ }^{8}$ abnormalities in platelet aggregation, ${ }^{9}$ anemia, ${ }^{10}$ and disorders of bone and mineral metabolism, including hyperphosphatemia, hypercalcemia, and secondary hyperparathyroidism. ${ }^{11}$ In observational studies of patients undergoing dialysis, elevated serum levels of phosphorus, calcium, parathyroid hormone, alkaline phosphatase, and fibroblast growth factor 23 (FGF23) have been associated with death and cardiovascular events. ${ }^{12-15}$ Disorders of mineral metabolism are thought to contribute to arterial calcification and diminished vascular compliance, ${ }^{16,17}$ contributing to myocardial ischemia, heart failure, and sudden death.

Cinacalcet (Sensipar/Mimpara, Amgen), a calcimimetic agent that acts by allosteric activation of the calcium-sensing receptor on parathyroid tissue, was approved for clinical use after its safety and efficacy in lowering levels of parathyroid hormone were shown in multiple randomized, controlled trials; levels of serum calcium and phosphorus were also consistently reduced..$^{18-20}$ In 2005 , in a study that pooled the results of three such trials of cinacalcet with a duration of 6 or more months, Cunningham et al. ${ }^{21}$ reported a reduction in the risk of cardiovascular events and fracture and a marked reduction in the rate of parathyroidectomy. The results of one trial suggested that cinacalcet attenuated the progression of vascular and cardiac-valve calcification. ${ }^{22}$

We designed the Evaluation of Cinacalcet Hydrochloride Therapy to Lower Cardiovascular Events (EVOLVE) trial to test the hypothesis that treatment with cinacalcet would reduce the risks of death and nonfatal cardiovascular events among patients with secondary hyperparathyroidism who were undergoing dialysis.

\section{METHODS}

\section{STUDY DESIGN}

In this multicenter, prospective, randomized, placebo-controlled trial, we compared cinacalcet with placebo in 3883 adults undergoing dialysis. All the patients were eligible to receive conventional therapy, including phosphate binders, vitamin D sterols, or both. The study design ${ }^{23}$ and the baseline characteristics of the patients ${ }^{24}$ have been reported previously. The study was approved by the institutional review board at each participating study site.

Randomization was stratified according to country and diabetes status with the use of fixed blocks. The sponsor, investigators, and patients were unaware of the treatment assignments.

\section{STUDY POPULATION}

Specific inclusion and exclusion criteria are listed in Table S1 in the Supplementary Appendix, available with the full text of this article at NEJM.org. Written informed consent was obtained from all patients.

\section{STUDY INTERVENTION}

After randomization, patients received either cinacalcet or placebo at a starting dose of $30 \mathrm{mg}$ daily. Patients were eligible for dose escalation once every 4 weeks during a 20-week escalation phase (to $60 \mathrm{mg}, 90 \mathrm{mg}, 120 \mathrm{mg}$, or $180 \mathrm{mg}$ daily) or every 8 weeks during follow-up, depending on levels of plasma parathyroid hormone and serum calcium. All patients received safety information regarding known risks and side effects of cinacalcet. Dialysis, phosphate binders, vitamin D sterols, calcium supplements, and other medications were prescribed at the discretion of the treating physicians, who were encouraged to adhere to published clinical-practice guidelines. Patients underwent laboratory testing, electrocardiography, and assessment of health-related quality of life according to the study design shown in Figure S1 in the Supplementary Appendix.

\section{STUDY END POINTS}

The primary composite end point was the time to death or the first nonfatal cardiovascular event (myocardial infarction, hospitalization for unsta- 
ble angina, heart failure, or a peripheral vascular event), as defined in detail in Table S2 in the Supplementary Appendix. Secondary end points included the time to the individual components of the primary composite end point, death from cardiovascular causes, stroke, bone fracture, and parathyroidectomy. All primary and secondary end points were adjudicated by an independent clinical-events classification group.

\section{BIOCHEMICAL MEASUREMENTS}

We measured plasma levels of parathyroid hormone and serum levels of calcium and phosphorus in central laboratories periodically throughout the trial. We performed an immunometric assay that detects the full-length peptide hormone along with various $\mathrm{N}$-terminally truncated parathyroid hormone fragments (as captured on intact parathyroid hormone assay).

\section{STUDY OVERSIGHT}

The study was sponsored by Amgen. An executive committee that was led by academic investigators supervised the trial design and operation, with representatives of Amgen as nonvoting members. An independent data monitoring committee reviewed safety data and interim analyses for efficacy. The sponsor collected the trial data and analyzed them according to a predefined statistical analysis plan. The analyses were verified by independent statisticians at Frontier Science, contracted by Amgen to support the EVOLVE data monitoring committee. The protocol and its amendments are available at NEJM.org.

The lead author wrote the first draft of the manuscript, and all coauthors provided substantive editing and approval. The executive committee made the decision to submit the manuscript for publication and takes full responsibility for the integrity of the data and interpretation of trial results and for the fidelity of this report to the study protocol.

\section{STATISTICAL ANALYSIS}

We calculated the proposed sample size on the basis of the following assumptions: an annual rate of the primary composite end point of $23.2 \%$ in the placebo group, a $20 \%$ treatment effect, a 1.5-year enrollment period, a 4-year total study duration, an annual rate of loss to follow-up of $1 \%$, an annual rate of dropout (withdrawal from active treatment before a primary event) of $10 \%$ in the cinacalcet group, and a rate of drop-in (use of commercially available cinacalcet before a primary event) of $10 \%$ in the placebo group. On the basis of a two-sided log-rank test for equality of survival functions, accounting for planned interim analyses with an overall alpha level of 0.05 , we determined that a primary event would need to occur in 1882 patients in order to ensure a power of approximately $90 \%$. After it became apparent that the overall (blinded) event rate was below $20.8 \%$, we extended the trial by 16 months to allow for accrual of the requisite number of events.

We collected and analyzed all end-point data in accordance with the intention-to-treat principle. For the time to the primary event, we computed Kaplan-Meier product-limit estimates of the eventfree survival time and compared groups using a two-sided log-rank test stratified according to country and diabetes status. We calculated relative hazards and 95\% confidence intervals from Cox proportional-hazards regression models, stratified according to country and diabetes status. We conducted prespecified multivariable analyses in which we adjusted for baseline characteristics. We used a closed-testing procedure to control the family-wise type I error rate at 0.05 between the primary composite end point and the secondary end points. The Hochberg procedure was prespecified to test significance among secondary end points. Since the primary end point was not significant, reported P values should be considered nominal.

We conducted prespecified companion analyses with lag censoring, in which data were censored 6 months after patients stopped using a study drug. We chose 6 months as the anticipated duration of any effect of altered mineral metabolism on extraskeletal calcification. Reasons for discontinuing a study drug before an end point included kidney transplantation, parathyroidectomy, and initiation of commercially available cinacalcet. We conducted companion analyses in which data were censored after these three events, alone or in combination. Finally, we compared event rates for all components of the primary composite end point using negative binomial regression and analyzed the time to multiple individual cardiovascular events, using the AndersenGill extension of the Cox model.

Data on adverse events were collected while 


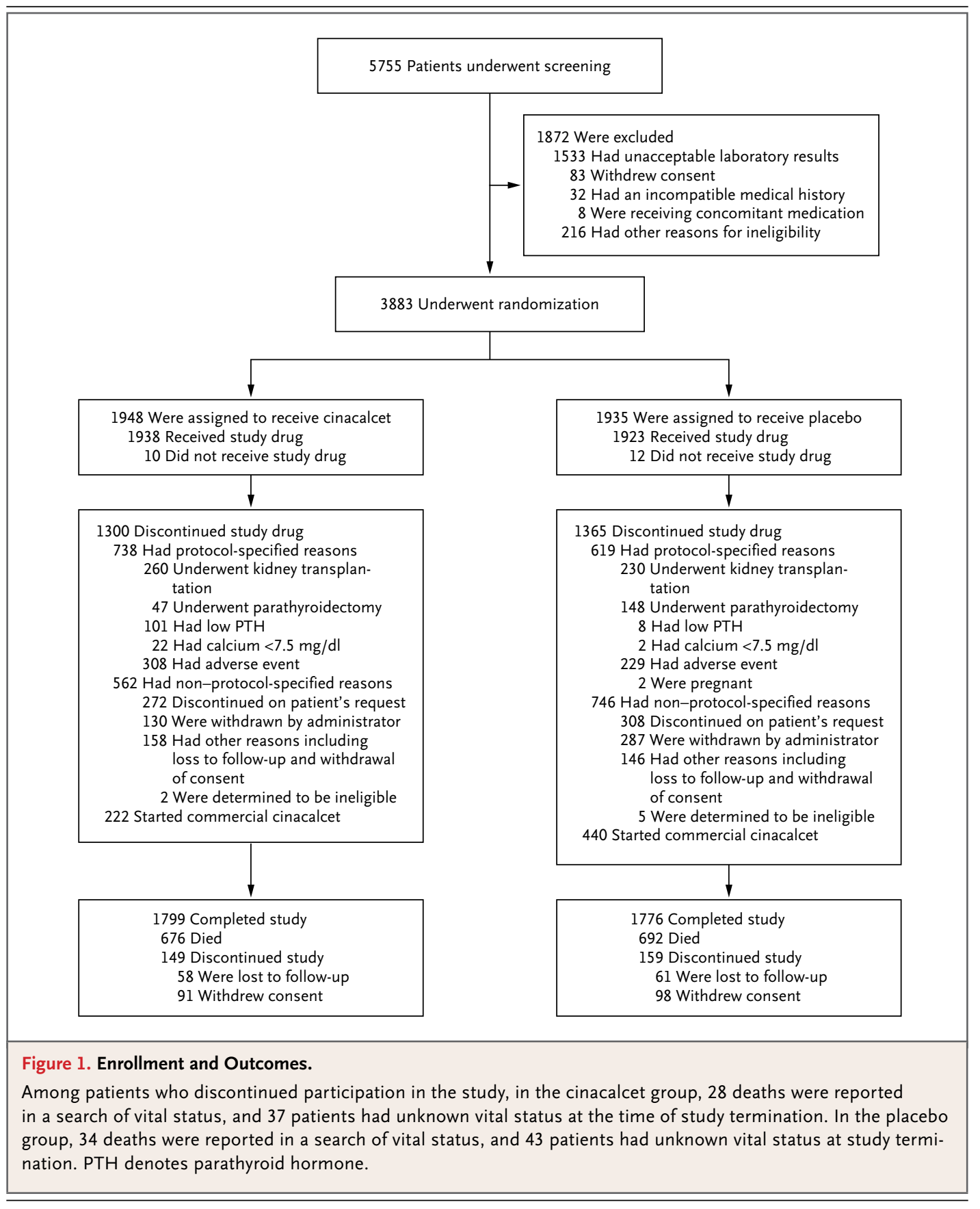

patients were taking a study drug. Three prespecified interim analyses were conducted by Frontier Science with the use of intention-to-treat data, with no censoring or adjustment (at approximately $25 \%, 50 \%$, and $75 \%$ of accrued events), resulting in a significance level of 0.044 for the final analysis. Statistical analyses were performed with the use of SAS software, version 9.2 (SAS Institute).

\section{RESULTS}

\section{PATIENTS}

From August 22, 2006, through January 31, 2008, a total of 3883 patients underwent randomization, including 1430 (36.8\%) in the United States, $1188(30.6 \%)$ in Europe, $687(17.7 \%)$ in Latin America, 283 (7.3\%) in Russia, 149 (3.8\%) in Aus- 


\begin{tabular}{|c|c|c|}
\hline Characteristic & $\begin{array}{l}\text { Cinacalcet } \\
(\mathrm{N}=1948)\end{array}$ & $\begin{array}{c}\text { Placebo } \\
(\mathrm{N}=1935)\end{array}$ \\
\hline \multicolumn{3}{|l|}{ Age (yr) } \\
\hline Median & 55.0 & 54.0 \\
\hline 10th to 90th percentile & $35.0-74.0$ & $35.0-73.0$ \\
\hline Female sex (\%) & 41.5 & 39.7 \\
\hline \multicolumn{3}{|l|}{ Race $(\%) \dagger$} \\
\hline White & 57.7 & 57.7 \\
\hline Black & 21.0 & 22.1 \\
\hline Other & 21.3 & 20.2 \\
\hline \multicolumn{3}{|l|}{ Body-mass indext } \\
\hline Median & 26.3 & 26.4 \\
\hline 10th to 90 th percentile & $20.4-36.4$ & $20.6-36.7$ \\
\hline \multicolumn{3}{|l|}{ Duration of dialysis (mo) } \\
\hline Median & 45.4 & 45.1 \\
\hline 10th to 90 th percentile & $8.5-142.0$ & $9.9-149.6$ \\
\hline \multicolumn{3}{|l|}{ Blood pressure (mm Hg) } \\
\hline \multicolumn{3}{|l|}{ Systolic } \\
\hline Median & 140 & 141 \\
\hline 10th to 90th percentile & $110-176$ & $111-177$ \\
\hline \multicolumn{3}{|l|}{ Diastolic } \\
\hline Median & 80 & 80 \\
\hline 10th to 90 th percentile & $60-100$ & $60-100$ \\
\hline \multicolumn{3}{|l|}{ Medical history (\%) } \\
\hline Diabetes & 33.6 & 33.5 \\
\hline Type 1 & 3.7 & 4.2 \\
\hline Type 2 & 29.8 & 29.4 \\
\hline Cardiovascular disease & 95.4 & 94.6 \\
\hline Hypertension & 92.5 & 91.7 \\
\hline Heart failure & 23.1 & 23.6 \\
\hline Peripheral vascular disease & 16.1 & 16.6 \\
\hline Coronary-artery bypass grafting & 6.9 & 8.0 \\
\hline Percutaneous coronary intervention & 6.7 & 6.8 \\
\hline Myocardial infarction & 12.3 & 12.6 \\
\hline Stroke & 8.3 & 10.0 \\
\hline Transient ischemic attack & 5.1 & 3.8 \\
\hline Amputation & 6.2 & 6.7 \\
\hline Atrial fibrillation & 10.4 & 11.6 \\
\hline
\end{tabular}

* There were no significant differences between the two groups except for mean diastolic blood pressure $(P=0.02)$ and transient ischemic attack $(P<0.05)$. $\uparrow$ Race was self-reported.

$\checkmark$ The body-mass index is the weight in kilograms divided by the square of the height in meters. tralia, and 146 (3.8\%) in Canada (Fig. 1). The trial population was diverse in terms of age, sex, and race or ethnic group; diabetes mellitus and underlying cardiovascular disease were relatively common (Table 1). A detailed description of baseline characteristics is provided in Table 3 in the Supplementary Appendix.

\section{STUDY TREATMENT}

The median duration of study-drug exposure was longer in the cinacalcet group than in the placebo group (21.2 months vs. 17.5 months). The daily median dose was $55 \mathrm{mg}$ (10th to 90th percentile, 28 to 130) for cinacalcet and $125 \mathrm{mg}$ (10th to 90th percentile, 43 to 161) for placebo. A total of $80.0 \%$ of patients in the placebo group reached the maximum daily dose, as compared with $38.3 \%$ of those in the cinacalcet group. Figure 2 shows the cumulative incidence of discontinuation of the study drug. Using the original assumption of a $20 \%$ treatment effect, but with the observed study duration and observed rates of events, dropout, and drop-in, which we assumed to be constant, we reestimated the statistical power to be 54\%.

Table S4 in the Supplementary Appendix provides additional details about study-drug discontinuation. In the placebo group, 384 of 1935 patients $(19.8 \%)$ began receiving commercially available cinacalcet before the occurrence of a primary event (corresponding to an annual rate of 7.4\%). In the cinacalcet group, 1207 of 1948 patients $(62.0 \%)$ discontinued the study drug, corresponding to an annual rate of $27.3 \%$. Although study-drug discontinuation was common, only $2.1 \%$ of patients were lost to follow-up during the study period of more than 5 years.

\section{INTENTION-TO-TREAT ANALYSIS}

\section{Primary Composite End Point}

The primary composite end point was reached in 938 of 1948 patients (48.2\%) in the cinacalcet group, as compared with 952 of 1935 patients $(49.2 \%)$ in the placebo group (relative hazard in the cinacalcet group vs. the placebo group, stratified according to country and diabetes status, 0.93; 95\% confidence interval [CI], 0.85 to 1.02 ; $\mathrm{P}=0.11$ ) (Fig. 3A). After adjustment for baseline characteristics, the relative hazard for the primary composite end point was 0.88 (95\% CI, 0.79 to $0.97 ; \mathrm{P}=0.008$ ) (Table $\mathrm{S} 5$ in the Supplementary 


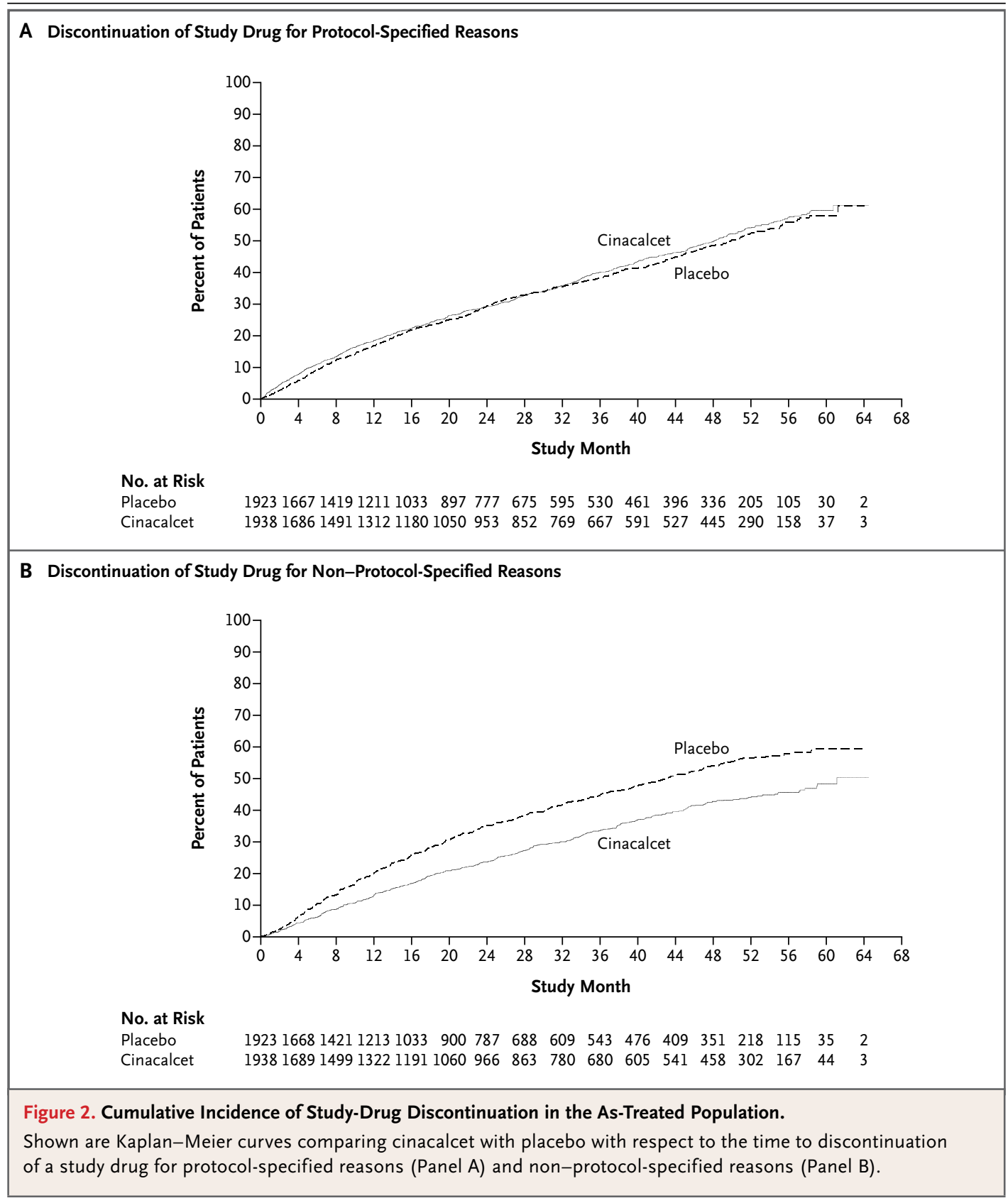

Appendix), and the relative hazard for death was 0.86 (95\% CI, 0.78 to $0.96 ; \mathrm{P}=0.006$ ).

The numbers of events for the components of the composite end point in the cinacalcet group were 703 deaths, 187 myocardial infarctions, 56 hospitalizations for unstable angina, 206 episodes of heart failure, and 184 peripheral vascular events. In the placebo group, the numbers were
718 deaths, 183 myocardial infarctions, 66 hospitalizations for unstable angina, 236 episodes of heart failure, and 200 peripheral vascular events. Relative hazards for the components of the composite end point minimally favored the cinacalcet group (Fig. 3B through 3F). Figure S2 in the Supplementary Appendix shows relative hazards for the primary composite end point stratified accord- 


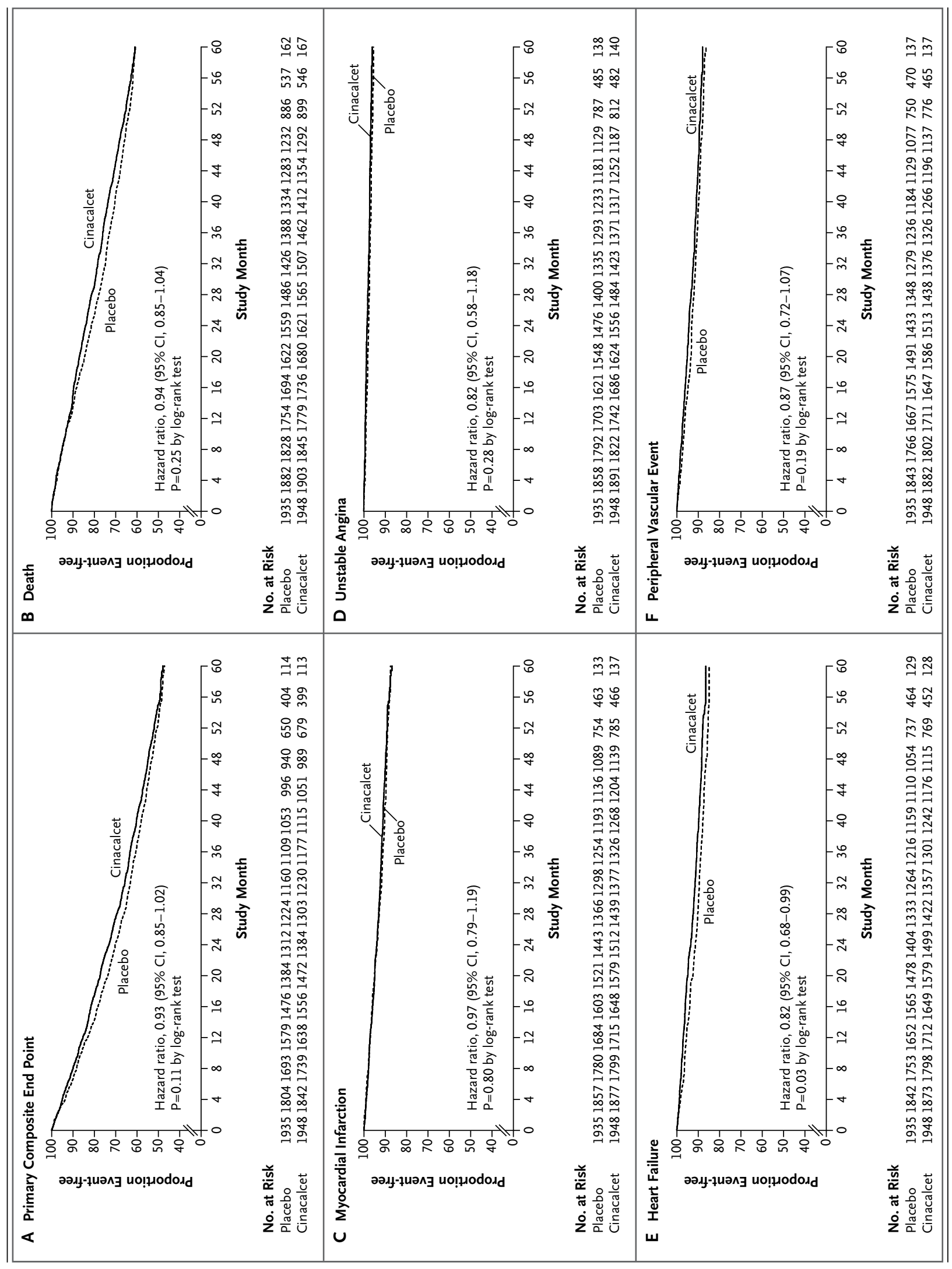


Figure 3 (facing page). Intention-to-Treat Analysis of the Primary Composite Outcome and Its Components.

Shown are Kaplan-Meier curves comparing cinacalcet with placebo for the time to the first primary composite outcome (Panel A), death (Panel B), first myocardial infarction (Panel C), first hospitalization for unstable angina (Panel D), first episode of heart failure (Panel E), and first episode of a peripheral vascular event (Panel F).

ing to prespecified baseline clinical characteristics. The effect of cinacalcet was more pronounced among older patients $(\mathrm{P}=0.03$ for interaction).

\section{Secondary End Points}

Stroke was adjudicated in 115 patients in the cinacalcet group as compared with 102 patients in the placebo group (relative hazard, 1.07; 95\% CI, 0.82 to $1.40 ; \mathrm{P}=0.61$ ). Death from cardiovascular causes was reported in 377 patients in the cinacalcet group and 391 in the placebo group (relative hazard, $0.92 ; 95 \% \mathrm{CI}, 0.80$ to $1.07 ; \mathrm{P}=0.28$ ). Parathyroidectomy and fracture results are shown in Text A in the Supplementary Appendix.

\section{Multiple Cardiovascular Events}

Myocardial infarction, hospitalization for unstable angina, heart failure, and peripheral vascular and stroke events per patient are shown in Figure S3 in the Supplementary Appendix. Cumulative event rates for the primary composite end point were 25.3 (95\% CI, 24.1 to 26.5) per 100 patientyears in the cinacalcet group and 27.3 (95\% CI, 26.0 to 28.5$)$ per 100 patient-years in the placebo group, a nominally significant result $(\mathrm{P}=0.02)$. There were no significant effects of cinacalcet on the risk of multiple cardiovascular events (Table S6 in the Supplementary Appendix).

\section{Biochemical Measures}

Median plasma levels of parathyroid hormone and serum levels of calcium, phosphorus, and the calcium-phosphorus product over time are shown in Figure S4 in the Supplementary Appendix. As expected, there was substantial group separation in levels of parathyroid hormone and calcium, which was maximal at approximately 4 months and narrowed over time.

\section{Concomitant Interventions}

The provision of antiplatelet agents, statins, betablockers, and inhibitors of the renin-angiotensinaldosterone system did not materially change over time in either group (Fig. S5 in the Supplementary Appendix).

\section{LAG-CENSORING ANALYSIS}

Censoring of data at 6 months after study-drug discontinuation yielded 638 primary composite end points in the cinacalcet group as compared with 658 in the placebo group (relative hazard, 0.85; 95\% CI, 0.76 to $0.95 ; \mathrm{P}=0.003$ ) (Fig. $4 \mathrm{~A}$ ). Figure 4 , panels $B$ through $F$, shows the components of the primary composite end point with the use of lag censoring; mortality was significantly reduced in the cinacalcet group (relative hazard, 0.83; $95 \%$ CI, 0.73 to $0.96 ; \mathrm{P}=0.009$ ). Table $\mathrm{S} 7$ in the Supplementary Appendix shows the results of an exploratory analysis comparing a range of lag durations used in calculating the relative hazard for the primary composite end point. Figure S6 in the Supplementary Appendix shows corresponding values for median levels of parathyroid hormone, calcium, phosphorus, and the calcium-phosphorus product; larger relative differences were sustained over time.

\section{OTHER SENSITIVITY ANALYSES}

When we censored data for patients after kidney transplantation, parathyroidectomy, or use of commercially available cinacalcet, relative hazards for the primary composite end point were 0.90 ( $95 \%$ CI, 0.82 to $0.99 ; \mathrm{P}=0.03$ ) for all three prespecified analyses. Censoring at the time of any of these three events yielded a relative hazard of 0.84 (95\% CI, 0.76 to $0.93 ; \mathrm{P}<0.001$ ). An inverse probability of censoring-weighted analysis is presented in Text B in the Supplementary Appendix.

\section{ADVERSE EVENTS}

Hypocalcemia developed in seven times as many patients in the cinacalcet group as in the placebo group, and nausea and vomiting were twice as common with cinacalcet (Table 2). Adverse effects led to discontinuation of the study drug in $18.1 \%$ of patients in the cinacalcet group and $13.0 \%$ of those in the placebo group. Rates of serious adverse events were similar in the two groups. Neoplastic events occurred in 115 patients in the cinacalcet group and 90 patients in the placebo group, corresponding to exposureadjusted rates of 2.9 and 2.5 events per 100 patient-years, respectively. Of the neoplastic events, 25 in the cinacalcet group and 23 in the placebo group were fatal (exposure-adjusted event rate, 


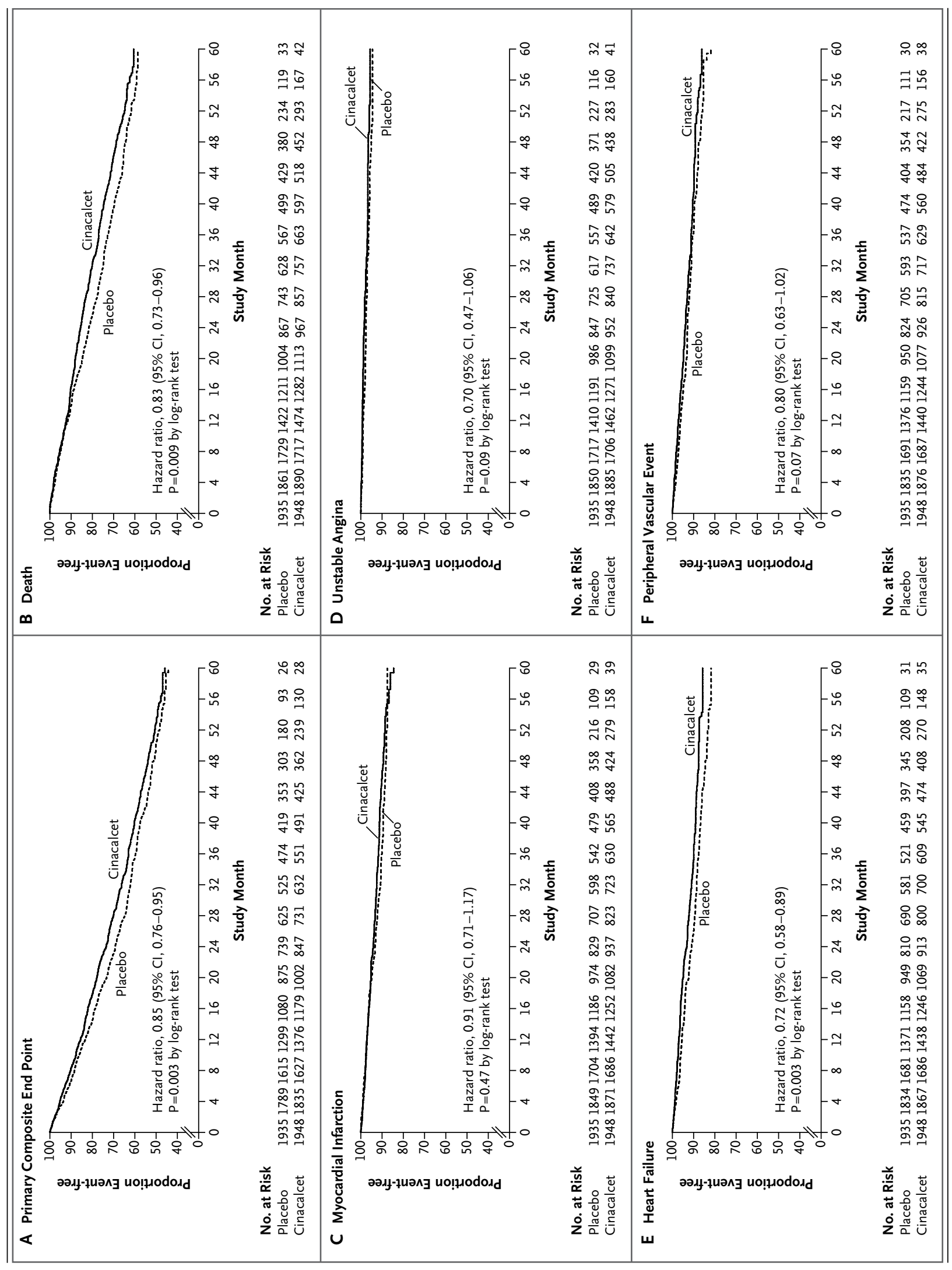


Figure 4 (facing page). Lag-Censoring Analysis of the Primary Composite Outcome and Its Components.

Shown are Kaplan-Meier curves comparing cinacalcet with placebo for the time to the first primary composite outcome (Panel A), death (Panel B), first myocardial infarction (Panel C), first hospitalization for unstable angina (Panel D), first episode of heart failure (Panel E), and first episode of a peripheral vascular event (Panel F).

0.6 per 100 patient-years in each group). Organspecific neoplastic events are listed in Table S8 in the Supplementary Appendix.

DISCUSSION

A limited number of interventions that are designed to enhance overall and cardiovascular health have been tested in patients undergoing dialysis. Normalization of the hematocrit with erythropoiesis-stimulating agents resulted in nominally higher rates of death, cardiovascular events, and vascular-access thrombosis. ${ }^{25}$ Higher-dose dialysis and high-flux dialysis membranes did not reduce the rates of death or cardiovascular events or improve nutritional status or health-related quality of life. ${ }^{26}$ Several trials of 3-hydroxy-3methylglutaryl coenzyme A (HMG-CoA) reductase inhibitors showed no significant benefits, ${ }^{27-29}$ despite consistently positive effects in the general population and in patients with chronic kidney disease not requiring dialysis. ${ }^{29-31}$

Secondary hyperparathyroidism has emerged as one of several complications associated with chronic kidney disease that might explain the exceptionally high rates of cardiovascular events and death among patients with end-stage renal disease. Several observational studies have shown significantly increased risks of death and cardiovascular events associated with levels of parathyroid hormone in excess of 600 pg per milliliter. ${ }^{12,13}$ Other studies have shown mixed results, ${ }^{14,32-34}$ with U-shaped, null, or inverse associations between the parathyroid hormone level and mortality, possibly confounded by nutritional status or inflammatory disease. No randomized, controlled trial has determined whether lowering the parathyroid hormone level reduces rates of death, cardiovascular events, or other major complications of mineral and bone disorders associated with chronic kidney disease.

In our study, there was a nonsignificant $7 \%$ reduction in the risk of the primary composite end point with cinacalcet in the intention-to-treat analysis. Given this result, the trial should be interpreted as nondefinitive. After adjustment for baseline characteristics, there was a nominally significant $12 \%$ reduction in risk. Other large randomized, controlled trials have shown more pronounced effect estimates after adjustment for unexpected differences in baseline determinants of risk. ${ }^{35-37}$ Although analyses accounting for studydrug exposure are subject to chance, bias, and confounding, we observed consistent (and nominally significant) effects, including a $15 \%$ reduction in the primary composite end point and a $17 \%$ reduction in mortality. Since parathyroidectomy or the initiation of treatment with commercially available cinacalcet would be expected to sharply reduce parathyroid hormone levels, differential application of these interventions after randomization would be expected to reduce the separation between groups and bias the estimate of the treatment effect toward the null.

The trial results must be interpreted in context. Patients undergoing dialysis are frequently frail and chronically ill. In the United States, mortality (20.7\% per year) and morbidity (median, 2 hospitalizations and 12 hospital days per year) are extraordinarily high for such patients, ${ }^{38}$ who commonly have cardiopulmonary, gastrointestinal, musculoskeletal, and neurocognitive symptoms, along with a median pill burden of 19 per day. ${ }^{39}$ Thus, recruitment for and retention in clinical trials is particularly challenging.

Cinacalcet reduced the rate of parathyroidectomy by more than half. The use of parathyroidectomy varied widely according to age, sex, and geographic region, with the lowest use in the United States and among the elderly. In an attempt to account for variation in the use of a surgical procedure for which there are no definitive indications, we used a conservative definition of severe, unremitting hyperparathyroidism that was based on available clinical-practice guidelines (Text A in the Supplementary Appendix). Combining data from patients who met biochemical criteria for severe, unremitting hyperparathyroidism with those from patients who actually underwent parathyroidectomy may provide a more accurate measure of secondary hyperparathyroidism that is refractory to conventional medical therapy.

Our study has several strengths. We enrolled patients from many geographic regions, who were 


\begin{tabular}{|c|c|c|c|c|c|c|}
\hline \multirow[t]{3}{*}{ Event } & \multicolumn{3}{|c|}{ Cinacalcet $(N=1938)$} & \multicolumn{3}{|c|}{ Placebo $(\mathrm{N}=1923)$} \\
\hline & $\begin{array}{l}\text { No. of } \\
\text { Patients }\end{array}$ & $\begin{array}{c}\text { Exposure- } \\
\text { Adjusted Rate } †\end{array}$ & $\begin{array}{c}\text { Crude } \\
\text { Incidencet: }\end{array}$ & $\begin{array}{l}\text { No. of } \\
\text { Patients }\end{array}$ & $\begin{array}{c}\text { Exposure- } \\
\text { Adjusted Rate† }\end{array}$ & $\begin{array}{c}\text { Crude } \\
\text { Incidenceł }\end{array}$ \\
\hline & & $\begin{array}{l}\text { no. of patients/ } \\
100 \text { patient- } y r\end{array}$ & $\%$ of patients & & $\begin{array}{l}\text { no. of patients/ } \\
100 \text { patient-yr }\end{array}$ & $\%$ of patients \\
\hline All adverse events』 & 1806 & 273.2 & 93.2 & 1748 & 217.8 & 90.9 \\
\hline Nausea』 & 563 & 18.3 & 29.1 & 299 & 9.1 & 15.5 \\
\hline Vomiting $\llbracket$ & 497 & 15.4 & 25.6 & 264 & 8.0 & 13.7 \\
\hline Diarrhea & 397 & 12.0 & 20.5 & 360 & 11.5 & 18.7 \\
\hline Serious adverse events & 1338 & 53.3 & 69.0 & 1351 & 56.9 & 70.3 \\
\hline \multicolumn{7}{|l|}{ Treatment-related events } \\
\hline Adverse events $\rrbracket$ & 890 & 35.3 & 45.9 & 363 & 11.3 & 18.9 \\
\hline Serious adverse events $q$ & 69 & 1.8 & 3.6 & 44 & 1.2 & 2.3 \\
\hline \multicolumn{7}{|l|}{ Events associated with important identified risk } \\
\hline Convulsions & 48 & 1.2 & 2.5 & 30 & 0.8 & 1.6 \\
\hline Hypocalcemia』 & 240 & 6.7 & 12.4 & 33 & 0.9 & 1.7 \\
\hline Hypersensitivity reaction & 183 & 4.9 & 9.4 & 160 & 4.6 & 8.3 \\
\hline \multicolumn{7}{|l|}{ Additional adverse events of interest } \\
\hline Acute pancreatitis & 20 & 0.5 & 1.0 & 20 & 0.5 & 1.0 \\
\hline Possibly drug-related hepatic disorder & 45 & 1.1 & 2.3 & 50 & 1.4 & 2.6 \\
\hline Nervous system disorder $\|$ & 711 & 24.3 & 36.7 & 586 & 20.5 & 30.5 \\
\hline Ventricular arrhythmia & 18 & 0.4 & 0.9 & 23 & 0.6 & 1.2 \\
\hline \multicolumn{7}{|l|}{ Neoplastic event'广 } \\
\hline Any & 115 & 2.9 & 5.9 & 90 & 2.5 & 4.7 \\
\hline Fatal & 25 & 0.6 & 1.3 & 23 & 0.6 & 1.2 \\
\hline Calciphylaxis $\|$ & 6 & 0.1 & 0.3 & 18 & 0.5 & 0.9 \\
\hline Hypercalcemia & 32 & 0.8 & 1.7 & 36 & 1.0 & 1.9 \\
\hline Hyperphosphatemia & 28 & 0.7 & 1.4 & 30 & 0.8 & 1.6 \\
\hline
\end{tabular}

* Listed are data for patients who received at least one dose of a study drug. P values were calculated for the exposure-adjusted incidence rate with the use of a two-sided normal approximation.

$\dagger$ The exposure-adjusted rate was calculated as 100 times the total number of patients who had a first event divided by the total number of patient-years of exposure. Exposure excludes gaps of more than 7 days between stopping and restarting of a study drug.

7 The crude incidence was calculated as 100 times the total number of patients who had an event divided by the number of patients who received at least one dose of a study drug.

$\int \mathrm{P}<0.001$.

व $P=0.049$.

$\| \quad \mathrm{P}<0.01$.

*** This category includes all patients with events reported in the system organ class of the Medical Dictionary for Regulatory Activities. Headache, dizziness, and paresthesia were the most commonly reported adverse events in this category.

†† Organ-specific neoplastic events are described in Table S7 in the Supplementary Appendix.

diverse in terms of age, race or ethnic group, and underlying kidney and cardiovascular disease. Patients who were assigned to receive either cinacalcet or placebo were all eligible to receive active therapy for mineral and bone disorders associated with chronic kidney disease, including phosphate binders and vitamin D ste- rols, along with frequent prescription of antihypertensive, antiplatelet, and lipid-lowering agents. All cardiovascular end points were independently adjudicated, and relatively few patients were lost to follow-up.

However, the study has several important limitations. The statistical power was hampered by a 
lower-than-anticipated event rate, which required a prolongation of follow-up time, a particularly problematic issue given the high rate of dropout related to trial fatigue, gastrointestinal side effects, and other factors. Although dropout was common in both groups, more patients in the cinacalcet group dropped out because of adverse effects. Although less frequent than dropout, the use of commercially available cinacalcet by nearly one in five patients in the placebo group also reduced the statistical power. We could not have anticipated the observed baseline imbalances between randomized groups, including a 1-year difference in age, the most potent predictor of death or cardiovascular events, which materially influenced the relative hazard and level of significance.
Analyses adjusted for baseline characteristics or taking into account the effects of parathyroidectomy, kidney transplantation, and ongoing studydrug use suggest that cinacalcet may result in nominally significant reductions in the risk of death or first myocardial infarction, hospitalization for unstable angina, heart failure, or a peripheral vascular event (relative reduction, 10 to $15 \%$; absolute reduction, 2 to 3 percentage points). However, in the unadjusted intention-to-treat analysis, as prespecified in the protocol, cinacalcet did not significantly reduce the risk of death or major cardiovascular events.

Supported by Amgen.

Disclosure forms provided by the authors are available with the full text of this article at NEJM.org.

APPENDIX

Members of the writing committee - Glenn M. Chertow, M.D., M.P.H., Stanford University School of Medicine, Palo Alto, CA; Geoffrey A. Block, M.D., Denver Nephrology, Denver; Ricardo Correa-Rotter, M.D., Instituto Nacional de Ciencias Médicas y Nutrición Salvador Zubirán, Mexico City; Tilman B. Drüeke, M.D., Picardie University School of Medicine and Pharmacy, Amiens, France; Jürgen Floege, M.D., Rheinisch-Westfälische Technische Hochschule Aachen University Hospital, Aachen, Germany; William G. Goodman, M.D., Amgen, Thousand Oaks, CA; Charles A. Herzog, M.D., University of Minnesota, Minneapolis; Yumi Kubo, M.S., Amgen, Thousand Oaks, CA; Gerard M. London, M.D., Hôpital Manhès, Paris; Kenneth W. Mahaffey, M.D., Duke Clinical Research Institute, Durham, NC; T. Christian H. Mix, M.D., Amgen, Thousand Oaks, CA; Sharon M. Moe, M.D., Indiana University School of Medicine, Roudebush Veterans Affairs Medical Center, Indianapolis; Marie-Louise Trotman, M.S., Amgen, Thousand Oaks, CA; David C. Wheeler, M.D., University College London, London; and Patrick S. Parfrey, M.D., Health Sciences Center, St. John's, NF, Canada — assume responsibility for the content and integrity of this article.

REFERENCES

1. Go AS, Chertow GM, Fan D, McCulloch CE, Hsu CY. Chronic kidney disease and the risks of death, cardiovascular events, and hospitalization. $\mathrm{N}$ Engl J Med 2004;351:1296-305.

2. Foley RN, Murray AM, Li S, et al. Chronic kidney disease and the risk for cardiovascular disease, renal replacement, and death in the United States Medicare population, 1998 to 1999. J Am Soc Nephrol 2005;16:489-95.

3. Nusair MB, Rajprohit N, Alpert MA Chronic inflammation and coronary atherosclerosis in patients with end-stage renal disease. Cardiorenal Med 2012;2: 117-24.

4. Himmelfarb J. Uremic toxicity, oxidative stress and hemodialysis as renal replacement therapy. Semin Dial 2009;22: 636-43.

5. Briet M, Burns KD. Chronic kidney disease and vascular remodeling: molecular mechanisms and clinical implications. Clin Sci (Lond) 2012;123:399-416.

6. Rubinger D, Backeroth R, Sapoznikov D. Sympathetic activation and baroreflex function during intradialytic hypertensive episodes. PLoS One 2012;7(5):e36943.

7. Mallamaci F, Tripepi G, Cutrupi S, Malatino LS, Zoccali C. Prognostic value of combined use of biomarkers of inflam- mation, endothelial dysfunction, and myocardiopathy in patients with ESRD. Kidney Int 2005;67:2330-7.

8. Jourde-Chiche N, Dou L, Cerini C, Dignat-Goerge F, Brunet P. Vascular incompetence in dialysis patients: proteinbound uremic toxins and endothelial dysfunction. Semin Dial 2011;24:327-37.

9. Mezzano D, Tagle $\mathrm{R}$, Panes $\mathrm{O}$, et al. Hemostatic disorder of uremia: the platelet defect, main determinant of the prolonged bleeding time, is correlated with indices of activation of coagulation and fibrinolysis. Thromb Haemost 1996;76: 312-21.

10. Fishbane S. Anemia and cardiovascular risk in the patient with kidney disease. Heart Fail Clin 2008;4:401-10.

11. Kidney Disease Improving Global Outcomes (KDIGO) CKD-MBD Work Group. KDIGO clinical practice guideline for the diagnosis, evaluation, prevention, and treatment of chronic kidney diseasemineral and bone disorder (CKD-MBD). Kidney Int Suppl 2009;76:S1-S130.

12. Block GA, Klassen PS, Lazarus JM, Ofsthun N, Lowrie EG, Chertow GM. Mineral metabolism, mortality and morbidity in maintenance hemodialysis. J Am Soc Nephrol 2004;15:2208-18.

13. Floege J, Kim J, Ireland E, et al. Serum
iPTH, calcium and phosphorus and the risk of mortality in a European haemodialysis population. Nephrol Dial Transplant 2011;26:1948-55.

14. Kalantar-Zadeh K, Kuwae N, Regidor $\mathrm{DL}$, et al. Survival predictability of timevarying indicators of bone disease in maintenance hemodialysis patients. Kidney Int 2006;70:771-80.

15. Gutiérrez OM, Mannstadt $M$, Isakova T, et al. Fibroblast growth factor 23 and mortality among patients undergoing hemodialysis. N Engl J Med 2008;359: 584-92.

16. Suzuki T, Yonemura K, Maruyama Y, et al. Impact of serum parathyroid hormone concentration and its regulatory factors on arterial stiffness in patients undergoing maintenance hemodialysis. Blood Purif 2004;22:293-7.

17. London GM, Marchais SJ, Guérin AP, Métivier F. Arteriosclerosis, vascular calcifications and cardiovascular disease in uremia. Curr Opin Nephrol Hypertens 2005;14:525-31.

18. Quarles LD, Sherrard DJ, Adler S, et al. The calcimimetic AMG 073 as a potential treatment for secondary hyperparathyroidism of end-stage renal disease. J Am Soc Nephrol 2003;14:575-83.

19. Block GA, Martin KJ, de Francisco AL, 
et al. Cinacalcet for secondary hyperparathyroidism in patients receiving hemodialysis. N Engl J Med 2004;350:151625.

20. Lindberg JS, Culleton B, Wong G, et al. Cinacalcet $\mathrm{HCl}$, an oral calcimimetic agent for the treatment of secondary hyperparathyroidism in hemodialysis and peritoneal dialysis: a randomized, double-blind, multicenter study. J Am Soc Nephrol 2005;16: 800-7.

21. Cunningham J, Danese $M$, Olson $\mathrm{K}$, Klassen P, Chertow GM. Effects of the calcimimetic cinacalcet $\mathrm{HCl}$ on cardiovascular disease, fracture, and healthrelated quality of life in secondary hyperparathyroidism. Kidney Int 2005;68: 1793-800.

22. Raggi P, Chertow GM, Block GA, et al. The ADVANCE study: a randomized trial to evaluate the effects of cinacalcet plus low dose vitamin $\mathrm{D}$ on vascular calcification in patients on hemodialysis. Nephrol Dial Transplant 2011;26:1327-39.

23. Chertow GM, Pupim LB, Block GA, et al. EVOLVE (Evaluation of Cinacalcet Therapy to Lower Cardiovascular Events): rationale and design of a randomized clinical trial. Clin J Am Soc Nephrol 2007; 2:898-905

24. Chertow GM, Correa-Rotter R, Block $\mathrm{GA}$, et al. Baseline characteristics of subjects enrolled in the Evaluation of Cinacalcet $\mathrm{HCl}$ Therapy to Lower Cardiovascular Events (EVOLVE) trial. Nephrol Dial Transplant 2012;27:2872-9.

25. Besarab A, Bolton WK, Browne JK, et al. The effects of normal as compared with low hematocrit values in patients with cardiac disease who are receiving hemodialysis and epoetin. N Engl J Med 1998;339:584-90.

26. Eknoyan G, Beck GJ, Cheung AK, et al. Effect of dialysis dose and membrane flux in maintenance hemodialysis. $N$ Engl J Med 2002;347:2010-9.

27. Wanner C, Krane V, März W, et al. Atorvastatin in patients with type 2 diabetes mellitus undergoing hemodialysis N Engl J Med 2005;353:238-48. [Erratum, N Engl J Med 2005;353:1640.]

28. Fellström BC, Jardine AG, Schmider $\mathrm{RE}$, et al. Rosuvastatin and cardiovascula events in patients undergoing hemodialysis. N Engl J Med 2009;360:1395-407. [Erratum, N Engl J Med 2010;362:1450.]

29. Baigent C, Landray MJ, Reith C, et al. The effects of lowering LDL cholesterol with simvastatin plus ezetimibe in patients with chronic kidney disease (Study of Heart and Renal Protection): a randomized placebo-controlled trial. Lancet 2011, 377:2181-92.

30. Tonelli M, Isles C, Curhan GC, et al Effect of pravastatin on cardiovascular events in people with chronic kidney disease. Circulation 2004;110:1557-63.

31. Nakamura H, Mizino K, Ohashi Y, et al. Pravastatin and cardiovascular risk in moderate chronic kidney disease. Atherosclerosis 2009;206:512-7.

32. Avram MM, Mittman N, Myint MM Fein P. Importance of low serum parathyroid hormone as a predictor of mortality in hemodialysis and peritoneal dialysis patients: 24 years of prospective observation. Am J Kidney Dis 2001;38:1351-7. 33. Melamed ML, Eustace JA, Plantinga L, et al. Changes in serum calcium, phosphate, and PTH and the risk of death in incident dialysis patients. Kidney Int 2006; 70:351-7.

34. Drechsler C, Krane V, Grootendorst DC, et al. The association between parathyroid hormone and mortality in dialysis patients is modified by wasting. Nephrol Dial Transplant 2009;24:3151-7.

35. Tavazzi L, Maggioni AP, Marchioli R, et al. Effect of $n-3$ polyunsaturated fatty acids in patients with chronic heart failure (the GISSI-HF trial): a randomized double-blind placebo-controlled clinical trial. Lancet 2008;372:1223-30.

36. O'Connor CM, Whellan DJ, Lee KL, et al. Efficacy and safety of exercise training in patients with chronic heart failure: HF-ACTION randomized controlled trial. JAMA 2009;301:1439-50.

37. Pfeffer MA, Swedberg K, Granger CB, et al. Effects of candesartan on mortality and morbidity in patients with chronic heart failure: the CHARM-Overall programme. Lancet 2003;362:759-66.

38. U.S. Renal Data System. USRDS 2011 annual data report: atlas of chronic kidney disease and end-stage renal disease in the United States. Bethesda, MD: National Institutes of Health, National Institute of Diabetes and Digestive and Kidney Diseases, 2011.

39. Chiu YW, Teitelbaum I, Misra M, de Leon EM, Adzize T. Mehrotra R. Pill burden, adherence, hyperphosphatemia and quality of life in maintenance hemodialysis patients. Clin J Am Soc Nephrol 2009; 4:1089-96.

Copyright @ 2012 Massachusetts Medical Society.

ICMJE SEEKING TWO NEW MEMBER JOURNALS

The International Committee of Medical Journal Editors (ICMJE) is seeking two new member journals to be represented by their editors-in-chief. Information about the ICMJE is available at www.icmje.org. Candidate journals should meet the following criteria:

- be a peer-reviewed general medical journal that publishes original research involving humans

- have a governance structure that ensures editorial independence

- have an editor with experience in the position who expects to continue in the position for at least another 3 years

- be financially able to support the editor's participation in ICMJE activities

In considering candidates, the ICMJE may seek to improve the balance of geographic areas and publishing models among its membership.

To apply, editors-in-chief of interested journals should submit the following materials to the ICMJE (at icmje@acponline.org):

- brief curriculum vitae

- cover letter describing the journal, including but not necessarily limited to details of the journal's history, sponsor or publisher, governance structure, publishing model (e.g., subscription, author-pays open access), target audience, print circulation and online traffic, number of manuscript submissions per year, processes used to select material for publication, acceptance rate, databases where indexed, website address, and guidelines for authors

- statement on how the journal might benefit from ICMJE membership and how the ICMJE might benefit from the journal's membership (should not exceed 1000 words)

The deadline for applications is January 31, 2013. 